\title{
Radiatively Inefficient Accretion Flow Models of Sgr A*
}

\author{
Eliot Quataert*1 \\ ${ }^{1}$ Astronomy Dept., 601 Campbell Hall, UC Berkeley, Berkeley, CA 94720
}

Received 15 November 2002, revised 30 November 2002, accepted 2 December 2002

Published online 3 December 2002

I review radiatively inefficient accretion flow models for the $\approx 2.6 \times 10^{6} M_{\odot}$ black hole $(\mathrm{BH})$ in the Galactic Center. I argue for a 'concordance model' of Sgr A*: both theory and observations suggest that hot ambient gas around the $\mathrm{BH}$ is accreted at a rate $\sim 10^{-8} M_{\odot} \mathrm{yr}^{-1}$, much less than the canonical Bondi rate. I interpret Chandra observations of Sgr A* in the context of such a model: (1) the extended 'quiescent' X-ray emission is due to thermal bremsstrahlung from gas in the vicinity of the Bondi accretion radius, and (2) the $\sim 10^{4}$ second long X-ray flares are due to synchrotron or Inverse-Compton emission by non-thermal electrons accelerated in the inner $\sim 10$ Schwarzschild radii of the accretion flow.

\section{Introduction}

The case for $\mathrm{a} \approx 2.6 \times 10^{6} M_{\odot}$ black hole $(\mathrm{BH})$ coincident with the radio source Sagittarius $\mathrm{A}^{*}$ in the Galactic Center (GC) is now compelling (e.g., Schödel et al. 2002; Ghez et al. 2003). This only emphasizes the long-standing puzzle that the luminosity from the $\mathrm{GC}$ is remarkably low given the presence of a massive black hole. The resolution of this puzzle must lie in how gas from the ambient medium accretes onto the central BH. In these proceedings I review accretion models and their application to Sgr A*.

A unique feature of the Galactic Center is our ability to constrain the dynamics of gas quite close to the black hole (relative to other systems), thus providing additional boundary conditions on, and much less freedom for, theoretical models. A canonical formulation of these constraints is the Bondi accretion estimate for the rate at which the BH gravitationally captures surrounding gas (Bondi 1952; see Melia 1992 for an early application to Sgr A*). Given relatively uniformly distributed matter with an ambient density $\rho_{0}$ and an ambient sound speed $c_{0}$, the sphere of influence of a $\mathrm{BH}$ of mass $M$ extends out to $R_{a c c} \approx G M / c_{0}^{2}$. The accretion rate of this gas onto the central $\mathrm{BH}$, in the absence of angular momentum and magnetic fields, is then $\dot{M}_{B} \approx \pi R_{a c c}^{2} \rho_{0} c_{0}$.

Chandra observations of the GC detect extended diffuse emission within $1-10^{\prime \prime}$ of the BH (Baganoff et al. 2003a). This emission likely arises from hot gas produced when the stellar winds from massive stars in the GC collide and shock (e.g., the He I cluster; Krabbe et al. 1991). Interpreted as such, the inferred gas density and temperature are $\approx 20 \mathrm{~cm}^{-3}$ and $\approx 1.3 \mathrm{keV}$ on $10^{\prime \prime}$ scales, and $\approx 100 \mathrm{~cm}^{-3}$ and $\approx 2$ $\mathrm{keV}$ on $\approx 1^{\prime \prime}$ scales (see also Fig. 3). The corresponding Bondi accretion radius is $R_{\text {acc }} \approx 0.04 \mathrm{pc} \approx 1^{\prime \prime}$ and the Bondi accretion rate is $\dot{M}_{B} \approx 10^{-5} M_{\odot} \mathrm{yr}^{-1} .{ }^{1}$ If gas were accreted at this rate onto the $\mathrm{BH}$ via a geometrically thin, optically thick accretion disk (Shakura \& Sunyaev 1973), a model that has been extensively and successfully applied to luminous accreting sources (e.g., Kortakar \& Blaes 1999), the expected luminosity would be $L \approx 0.1 \dot{M}_{B} c^{2} \sim 10^{41} \mathrm{ergs} \mathrm{s}^{-1}$, larger than the observed luminosity by a factor of $\sim 10^{5}$. This is the strongest argument against a thin disk in Sgr $\mathrm{A}^{*}$. An additional argument is the absence of any disk-like blackbody emission component in the spectrum of $\mathrm{Sgr} \mathrm{A}^{*}$. If the putative

\footnotetext{
* e-mail: eliot@astron.berkeley.edu; Phone: 510-642-3792

1 This is much less than the total mass loss rate from stars in the GC $\left(\approx 10^{-3} M_{\odot} \mathrm{yr}^{-1}\right.$; Najarro et al. 1997), implying that there should also be a global outflow of hot gas from the central parsec (a GC 'wind')
} 
disk were to extend all the way down to the $\mathrm{BH}$, its accretion rate would have to be $\lesssim 10^{-10} M_{\odot} \mathrm{yr}^{-1} \approx$ $10^{-5} \dot{M}_{B}$ to satisfy infrared limits (e.g., Narayan 2002; see his Fig. 2).

One possible caveat to the Bondi analysis is that there is far more (by mass) cold molecular gas than hot X-ray emitting gas in the central $1-10$ parsecs of the GC (e.g., Herrnstein \& Ho 2002). It is unclear how close to the $\mathrm{BH}$ the molecular gas extends and whether it is important for the dynamics of gas accreting onto the BH. In what follows I ignore this component, but see Nayakshin (2003) for a different view.

As emphasized above, the inferred low efficiency of Sgr A* is the strongest argument against accretion proceeding via a thin accretion disk. Instead, the observations favor models in which very little of the gravitational potential energy of the inflowing gas is radiated away. I will refer to such models as radiatively inefficient accretion flows (RIAFs). In the next section ( $(2)$ I summarize the properties of RIAFs. I then apply these models to the GC ( 33$)$, emphasizing the interpretation of radio and X-ray observations of Sgr $A^{*}$. Finally, I conclude with a brief summary $(\S 4)$.

\section{Radiatively Inefficient Accretion Flows}

RIAFs describe the dynamics of rotating accretion flows in which $L \ll 0.1 \dot{M} c^{2}$, i.e., very little energy generated by accretion is radiated away (e.g., Ichimaru 1977; Rees et al. 1982; Narayan \& Yi 1994). Instead, the gravitational potential energy released by turbulent stresses in the accretion flow is stored as thermal energy. As a result, the accreting gas is very hot, with a characteristic thermal energy comparable to its gravitational potential energy; close to the $\mathrm{BH}$ this implies $T \sim G M m_{p} / 3 k R \sim 0.1 m_{p} c^{2} / k \sim 10^{12}$ $\mathrm{K}$. At such temperatures, and for gas densities appropriate to systems like the GC, the Coulomb collision time is much longer than the time it takes gas to flow into the BH. The accretion flow then develops a twotemperature structure with the protons likely hotter than the electrons: $T_{p} \sim 10^{12} \mathrm{~K} \gtrsim T_{e} \sim 10^{10}-10^{12}$ $\mathrm{K}$. The precise electron temperature is uncertain but important since electrons produce the radiation that we see. The electron temperature depends on how and to what extent they are heated by processes such as shocks, MHD turbulence, and reconnection (see, e.g., Quataert \& Gruzinov 1999). Note that because collisions are unimportant one would not expect the electron distribution function to be thermal.

Advection-Dominated Accretion Flows (ADAFs) are a simple analytical model for the dynamics of RIAFs; they predict that the structure of the flow is in some ways similar to spherical Bondi accretion, despite the fact that angular momentum and viscosity are important (e.g., Ichimaru 1977; Narayan \& Yi 1994). In ADAF models the gas rotates at $\Omega \approx 0.3-0.5 \Omega_{K}$, where $\Omega_{K}=\sqrt{G M / R^{3}}$ is the Keplerian rotation rate. Because the flow is hot, pressure forces are also important and the inflowing gas is geometrically quite "thick," with a scale height $H \approx R$ at every radius. The radial velocity in the flow is given by $v_{R} \approx \alpha c_{s} \approx \alpha v_{K}$ where $\alpha$ is the dimensionless viscosity parameter, $c_{s}$ is the sound speed in the flow and $v_{K}=R \Omega_{K} \propto R^{-1 / 2}$. Conservation of mass on spherical shells then implies that the density scales as $\rho \propto R^{-3 / 2}$, the characteristic scaling for spherical accretion. ADAF models also predict that, even in the presence of rotation, the rate at which gas accretes onto the $B H$ from an ambient medium is comparable to the Bondi accretion rate: $\dot{M}_{A D A F} \sim \dot{M}_{B} .{ }^{2}$ Thus in ADAF models the low luminosity of $\operatorname{Sgr} A^{*}$ is due to a very low radiative efficiency $\sim 10^{-6}$.

With the advent of global, time-dependent, numerical simulations of accretion flows, it has become possible to numerically simulate RIAFs and test the ADAF predictions. Note that RIAFs are, in one sense, the easiest flows to simulate since (1) no treatment of radiation or radiative transfer is needed and (2) the flow is "thick" with $H \sim R$, so there is no difficult-to-simulate separation of scales like in a thin disk. On the other hand, for a system like the GC, the proton-electron collision time close to the BH is $\sim 6$ orders of magnitude longer than the inflow time of the gas. Thus the fluid approximation used by all simulations

${ }^{2}$ A more accurate estimate might be $\dot{M}_{A D A F} \approx \alpha \dot{M}_{B}$ (e.g., Narayan 2002). The factor of $\alpha$ arises because the inflow velocity of gas at the Bondi accretion radius is $\approx \alpha c_{s}$ in ADAF models while it is $\approx c_{s}$ in Bondi models. Thus for a fixed density in the ambient medium, the accretion rate in an ADAF will be smaller by a factor of $\alpha$. 
to date is suspect (a kinetic treatment should be used; see Quataert et al. 2002). Whether this introduces qualitative or merely quantitative errors in the results is unknown. I suspect the latter.

The key result from nearly all simulations to date is that $\dot{M} \ll \dot{M}_{B}$, i.e., very little mass available at large radii actually accretes onto the black hole (e.g., Stone, Pringle, \& Begelman 1999; Igumenshchev \& Abramowicz 1999; 2000; Igumenshchev et al. 2000; Stone \& Pringle 2001; Hawley \& Balbus 2002; Igumenshchev et al. 2003). Another way to state this result is that the radial density profile in the flow is much "flatter" than the ADAF predictions: for a given gas density at large distances from the black hole (e.g., measured by Chandra on 1" scales in the GC), the density close to the BH is much less than the ADAF or Bondi predictions. Following a proposal due to Blandford \& Begelman (1999), we can parameterize the density profile of RIAFs with a parameter $p$, where $\rho \propto R^{-3 / 2+p}$. With this parameterization, the rate at which gas is actually accreted into the $\mathrm{BH}$ is $\sim\left(R_{\text {in }} / R_{\text {out }}\right)^{p} \dot{M}_{B}$, where $R_{\text {in }} \sim R_{S}$ is the inner radius of the flow and $R_{\text {out }} \sim R_{\text {acc }}$ is the outer radius. Values of $p \approx 1 / 2-1$, rather than $p=0$ predicted by ADAF models, are favored by the simulations. This implies that, within the context of RIAF models, a low accretion rate, $\dot{M} \ll \dot{M}_{B}$, rather than just a low efficiency, is a major factor in the faintness of Sgr $A^{*}$.

Since the focus of this review is the application of RIAF models to Sgr A*, I will not dwell on why the simulations differ so significantly from the ADAF predictions. A brief discussion is, however, in order. The following material will not be used in later sections so uninterested readers can move directly to $\S 3$.

In RIAFs, the inflowing gas is heated at a rate $\approx 0.1 \dot{M} c^{2}$, as required by the release of gravitational potential energy in a differentially rotating accretion flow. In ADAF models this energy is stored as thermal energy and carried into the BH. As noted above, the inflowing gas is then very hot with a sound speed at any radius comparable to the escape speed from the BH's potential well. ADAF models are therefore prone to developing outflows (see Narayan \& Yi 1994 or Blandford \& Begelman 1999 for a more formal discussion). This led Blandford \& Begelman (1999) to propose that, in the absence of radiation, the gravitational binding energy of the accreted gas must be lost through some other (non-radiative) means. Otherwise the inflowing gas is not sufficiently bound to the BH to accrete. The numerical simulations to date are broadly consistent with this hypothesis; e.g., the gas temperature in the simulations is generally a factor of few-5 less than in ADAF models implying that the gas is indeed more strongly bound to the $\mathrm{BH}$.

The non-radiative energy loss can take one of two forms: (1) efficient turbulent transport of energy through the accretion flow to large radii or (2) a global outflow ('wind') that carries away the binding energy of the accreted matter. In hydrodynamic simulations (e.g., Stone et al. 1999; Igumenshchev \& Abramowicz 1999) or in MHD simulations with relatively 'weak' magnetic fields ( $\beta \gtrsim 10-100$, where $\beta$ is the ratio of the gas pressure to the magnetic pressure), convective transport of energy and angular momentum dominates the dynamics of the accretion flow (Narayan et al. 2002; Igumenshchev et al. 2003; see Quataert \& Gruzinov 2000a and Narayan et al. 2000 for such 'convection-dominated accretion flow' models). The convective luminosity through a spherical shell at radius $R$ is $\propto \rho v_{c}^{3} R^{2}$ where $v_{c}$ is the turbulent convective velocity. Since the flow is hot, $v_{c} \propto c_{s} \propto v_{K} \propto R^{-1 / 2}$. A constant flow of gravitational binding energy from small to large radii therefore requires $\rho \propto R^{-1 / 2}$; i.e., $p=1$ instead of $p=0$ as in ADAF models. ${ }^{3}$

In contrast to the hydrodynamic results, in MHD simulations with strong magnetic fields $(\beta \lesssim 10)$, MHD turbulence dominates the flow dynamics and convection is unimportant (e.g., Stone \& Pringle 2001; Hawley \& Balbus 2002; Igumenshchev et al. 2003). Stone \& Pringle (2001) and Hawley \& Balbus (2002) find that most of the inflowing gas is lost to a magnetically driven wind. Igumenshchev et al. (2003), on the other hand, find a much more complex flow configuration, though still with a very small accretion rate.

\footnotetext{
3 The convective energy flux could launch a thermally driven wind from large radii $\sim R_{a c c}$ or from the surface layers of the accretion flow. Thus there is not necessarily a clean distinction between global energy transport by turbulence and mass outflow as mechanisms for 'non-radiative' energy loss. Both processes are related and, in particular, the former can drive the latter.
} 


\section{RIAF Models Applied to Sgr A*}

A number of authors have used RIAF models, and in particular ADAF models, to explain the observed properties of Sgr A* (e.g., Narayan et al. 1995, 1998; Manmoto et al. 1997). These calculations have shown that an ADAF model accreting at the observationally inferred rate, $\dot{M} \sim \alpha \dot{M}_{B}$, can roughly account for the observed luminosity and spectrum of Sgr A*. The key constraint is that the fraction of the turbulent energy that heats the electrons, $\equiv \delta$, must be small $\lesssim 0.01$, so as to not overproduce the observed luminosity. Equivalently the electron temperature close to the $\mathrm{BH}$ must be $\lesssim 10^{10} \mathrm{~K} \ll T_{p} \approx 10^{12} \mathrm{~K}$. An example of such a model in shown by the dotted line in Figure 1 The model roughly reproduces the observed sub-mm emission, satisfies the IR limits, and produces an X-ray luminosity comparable to that seen by Chandra in the quiescent (non-flaring) state (I discuss the Chandra observations in more detail below). It does, however, significantly underproduce the lower frequency radio emission. Since the lower frequency radio emission in Sgr A* is phenomenologically similar to that of other AGN, a natural interpretation is that a jet is present and produces the radio emission (e.g., Falcke \& Markoff 2000, Yuan et al. 2001, and references therein). Alternatively, the results in Figure 1 assume purely thermal electrons, for which there is no good justification. As Figure 2 shows, even a small population of nonthermal electrons in the accretion flow can produce the radio emission (e.g., Mahadevan 1998; Ozel et al. 2000).

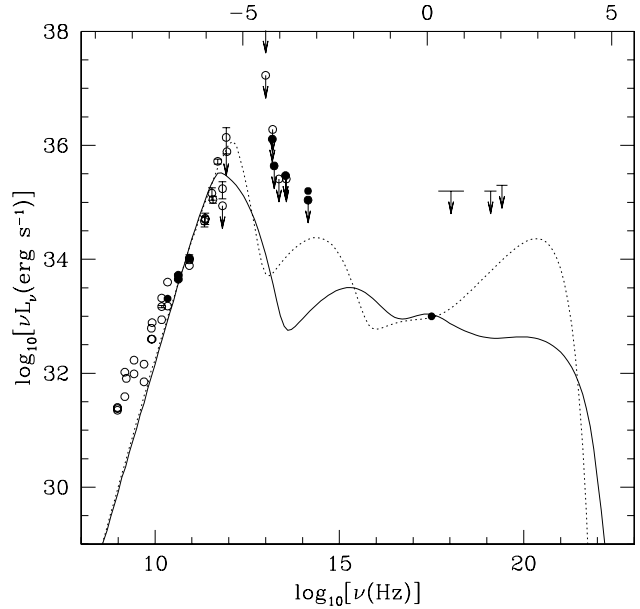

Fig. 1 Two 'baseline' RIAF models of Sgr A* that reproduce the quiescent Chandra flux (the Chandra spectrum is discussed in Figs 2 and 3. Dotted line: a RIAF model with $p=0, \dot{M} \approx \dot{M}_{B}$ and $\delta=0.01$ ( $\delta \equiv$ fraction of turbulent energy heating electrons). This is an ADAF-type model. Solid line: a RIAF with $p=0.5$ and a net accretion rate into the $\mathrm{BH}$ of $\dot{M} \approx 10^{-8} M_{\odot} \mathrm{yr}^{-1} \ll \dot{M}_{B} ; \delta=0.5$. This Figure is based on Quataert \& Narayan (1999).

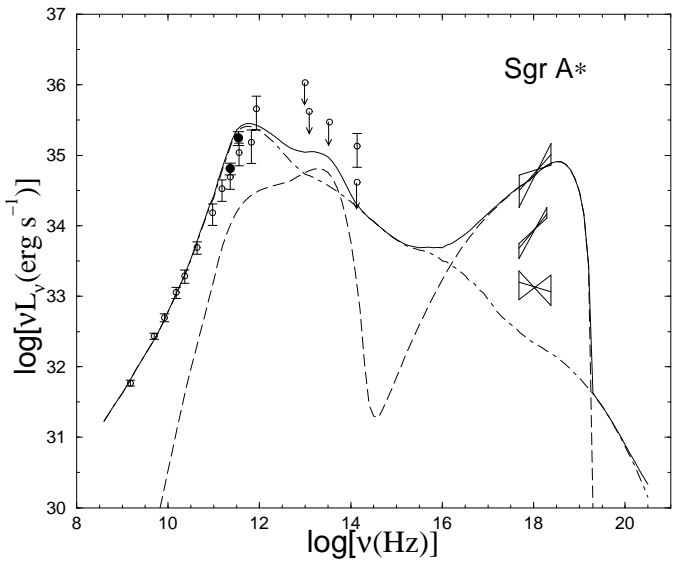

Fig. 2 Dot-dashed: A RIAF with $p \approx 0.4$ and $\delta \approx 0.5$. There is also a power law distribution of electrons with $n(\gamma) \propto \gamma^{-3.5}$ and $\approx 2 \%$ of the electron thermal energy. Power-law electrons produce the low freq. radio emission not accounted for in Fig. 1 Dashed line: Inverse-Compton model for the Chandra X-ray flare (see text for details). Solid line: Total emission. X-ray error bars are (from top to bottom): Oct. 2000 flare, average flare, \& quiescent emission. This Figure is based on Yuan et al. (2003).

Quataert \& Narayan (1999) showed that a much broader class of RIAF models could also account for the observed properties of Sgr A*. Specifically, the low accretion rate models favored theoretically $(\S 2)$ can reproduce the observations as well. The key requirement is that the electrons must be hotter so as to produce more emission even though $\dot{M}$ and the gas density are lower. An example of such a model is shown by the solid line in Figure $1 p=0.5$ from $R_{\text {out }}=10^{5} R_{S}$ down to $R_{\text {in }}=R_{S}$, implying 
that the accretion rate into the $\mathrm{BH}$ is much smaller than the Bondi rate $\left(\sim 10^{-8} M_{\odot} \mathrm{yr}^{-1}\right)$. The electron temperature close to the $\mathrm{BH}$ is $\sim 10^{11} \mathrm{~K}$, rather than $\sim 10^{10} \mathrm{~K}$ as in ADAF models.

To first approximation, the two models in Figure1 1 reproduce the observed spectrum of Sgr A* equally "well" (or poorly, depending on one's vantage point). However, Aitken et al. (2000) and Bower et al.'s (2003) detection of $\approx 10 \%$ linear polarization in the sub-mm $(230 \mathrm{GHz})$ emission from $\mathrm{Sgr}$ A* argues strongly for low accretion rate models with $\dot{M} \lesssim 10^{-8} M_{\odot} \mathrm{yr}^{-1}$.

Beckert \& Falcke (2002, 2003; see also Ruszkowski \& Begelman 2002) present detailed models for the radio polarization of $\mathrm{Sgr} \mathrm{A}^{*}$ (both linear and circular). Here I summarize the constraints imposed by the linear polarization detection: accretion at $\sim \dot{M}_{B}$ implies a much higher gas density and magnetic field strength close to the black hole than accretion at $\ll \dot{M}_{B}$. Faraday rotation is therefore much stronger (e.g., Quataert \& Gruzinov 2000b; Agol 2000). In models with $\dot{M} \sim \dot{M}_{B}$, the rotation measure is $\gtrsim$ $10^{10} \mathrm{rad} \mathrm{m}^{-2}$ in the region of the flow $\left(\lesssim 10-100 R_{S}\right)$ where the sub-mm emission is produced. This leads to a Faraday rotation angle $\sim 10^{5} \mathrm{rad}$ at $\sim 100 \mathrm{GHz}$. This large rotation angle implies that intrinsically linearly polarized synchrotron emission would be depolarized propagating to the observer over most of the radio to infrared spectrum. By contrast, Bower et al. (2003) find that $R M \lesssim 10^{6} \mathrm{rad} \mathrm{m}^{-2}$ in their linear polarization detection at $230 \mathrm{GHz}$. Models with $\dot{M} \ll \dot{M}_{B}$ can satisfy this constraint because the density and magnetic field strength close to the BH are much smaller. For example, for $\dot{M} \approx 10^{-8} M_{\odot} \mathrm{yr}^{-1}$, the net rotation measure through the accretion flow is $R M \approx 10^{6} \mathrm{rad} \mathrm{m}^{-2}$, consistent with the observational constraint. Thus the observed detection of linear polarization in the sub-mm emission of Sgr $\mathrm{A}^{*}$ argues for a low accretion rate $\lesssim 10^{-8} M_{\odot} \mathrm{yr}^{-1} \ll \dot{M}_{B}$. One way out of this conclusion is to posit that the magnetic field undergoes so many reversals along the line of sight that the net Faraday rotation is $\gtrsim 10^{5}$ times smaller than these simple estimates (Ruszkowski \& Begelman 2002). I regard this as very unlikely, but am not aware of a direct observational argument against this possibility at the present time.

In light of the above arguments the rest of my discussion centers on models with $\dot{M} \ll \dot{M}_{B}$ : these 'concordance' models are both theoretically favored and satisfy the rotation measure constraint from the linear polarization detection. I focus on interpreting the Chandra X-ray observations of Sgr A*. Much of this material is based on Quataert (2002) and Yuan, Quataert, \& Narayan (2003).

Chandra observations reveal that there are two components to the X-ray emission coincident with Sgr A* (Baganoff et al. 2001, 2003ab): (1) a 'baseline' X-ray flux with a nearly constant luminosity $\approx 2 \times 10^{33}$ $\operatorname{ergs~s}^{-1}$ and a soft spectrum (photon index $\Gamma \approx 2.7$, where $\nu L_{\nu} \propto \nu^{2-\Gamma}$ ). This component is clearly extended with a size of $\approx 1^{\prime \prime} \approx 10^{5} R_{S}$ and does not vary in time. (2) X-ray 'flares' occurring at a rate of $\approx 1$ day $^{-1}$ and lasting $\approx 10^{3}-10^{4} \mathrm{~s}$. The luminosity increases by a factor of few -100 during the flare and the spectrum is quite hard $(\Gamma \approx 1.2)$. The flares are not extended; in fact, the observed timescales argue that they arise close to the $\mathrm{BH}$, at $\lesssim 10-100 R_{S}$.

\subsection{X-ray flares}

The X-ray flares are the most dramatic result from the Chandra observations. Markoff et al. (2001) showed that the flares are probably due to electron heating or acceleration, rather than a change in the accretion rate onto the $\mathrm{BH}$ (otherwise there is too much variation in other wavebands). An obvious analogue is magnetic reconnection in solar flares, which one could readily imagine occurring in the inner part of the accretion flow close to the $\mathrm{BH}$. Given an injection of energy into the electrons, there are three emission mechanisms that could, a priori, give rise to flares: (1) bremsstrahlung, (2) synchrotron, and (3) inverse Compton.

Bremsstrahlung is attractive because it can naturally explain the very hard spectrum of the flares. The problem is that, to produce a luminosity of $L_{35} 10^{35} \mathrm{ergs} \mathrm{s}^{-1}$ from a sphere of radius $R$, the gas density must be $n \approx 10^{9} L_{35}^{1 / 2} T_{e, 10}^{1 / 4}\left(R / 10 R_{S}\right)^{-3 / 2} \mathrm{~cm}^{-3}$, where $T_{e, 10}=T_{e} / 10^{10} \mathrm{~K}$. For comparison, the ambient density in the inner $10 R_{S}$ for a model with $\dot{M} \approx 10^{-8} M_{\odot} \mathrm{yr}^{-1}$ is $\approx 10^{6} \mathrm{~cm}^{-3}$. Equally importantly, bremsstrahlung emission in RIAFs is dominated by very large radii $\sim R_{a c c}$, not small radii (e.g., Quataert \& Narayan 1999; see below). Thus bremsstrahlung appears unlikely to be responsible for the X-ray flares. 
Two possible ways out of this conclusion are (1) to posit that the gas density increases by a factor of $\sim 10^{3}$ during the flare, and does so preferentially in the very inner part of the accretion flow (e.g., Liu \& Melia 2002). It is unclear, however, what would drive such large density changes, particularly since the cooling time is so long that thermally driven instabilities are unlikely to be important, (2) perhaps the accreting gas is a two-phase medium, with a cooler, denser phase giving rise to the X-ray flares (Yuan et al. 2003).

In contrast to bremsstrahlung, synchrotron emission can readily account for the observed flares: if $\sim 10 \%$ of the electrons (by energy) are accelerated into a power-law tail in the inner $\sim 10 R_{S}$, the radio-IR emission is essentially unchanged while electrons with Lorentz factors $\gamma \sim 10^{5}$ can produce the X-ray emission (e.g., Markoff et al. 2001). Moreover, there is a 'natural' explanation for the hard X-ray spectrum seen. For RIAF models with $\dot{M} \approx 10^{-8} M_{\odot} \mathrm{yr}^{-1}$, the magnetic field strength close to the $\mathrm{BH}$ is $\approx 20 B_{20}$ G. The associated synchrotron cooling time for electrons emitting in the Chandra band is $\approx 20 B_{20}^{-3 / 2}$ s. Thus, unless $B \lesssim 0.3 \mathrm{G}$ in the emitting region, the cooling time is less than the duration of the flare and there should be a cooling break in the electron distribution function below the Chandra band. For an injected distribution of power-law electrons with $p_{e}<2$, where $n(\gamma) \propto \gamma^{-p_{e}}$, the distribution function for the population of cooled electrons is $n(\gamma) \propto \gamma^{-2}$. This implies a synchrotron spectrum with $\Gamma=1.5$, consistent with the typical flare observed by Chandra (Baganoff et al. 2003b).

Synchrotron self-Compton emission can also explain the X-ray flares observed by Chandra. The dashed line in Figure 2 shows a concrete example in which most of the electrons in a $\approx 3 R_{S}$ volume are accelerated into a power law distribution. This population of electrons produces synchrotron emission and also upscatters synchrotron photons to produce a hard X-ray flare. Note that there is very little change to the radio or IR emission (the dot-dashed line in Fig. 2] shows the baseline non-flaring model in which power-law electrons have only $\approx 2 \%$ of the electron thermal energy).

Finally, it is important to stress that in the models discussed here, the duration of the flare is set by a dynamical or viscous timescale in the inner $\sim 10 R_{S}$ of the accretion flow. By contrast, there is no explanation for the mean time between flares, $\approx 1$ day. In addition, it is difficult to apply the ideas considered here to the week-long, factor of few, sub-mm 'flares' observed by Tsuboi et al. (1999) and Zhao et al. (2003). In particular the duration of these 'flares' is inconsistent with the characteristic timescales in the sub-mm emitting region $\left(\lesssim 10 R_{S}\right)$. One possibility is that they are not directly related to the Chandra flares but are instead due to small fluctuations in $\dot{M}$ set by dynamics in the accretion flow at larger radii.

\subsection{Quiescent X-ray Emission}

The steady quiescent emission observed by Chandra is qualitatively different from the flaring emission. In particular, it is softer $(\Gamma \approx 2.7)$ and extended $\left(\approx 1^{\prime \prime} \approx 10^{5} R_{S}\right)$. The latter fact implies that it is a different emission component since synchrotron and inverse Compton emission are produced at small radii.

RIAF models naturally predict that the thermal bremsstrahlung emission is dominated by large radii in the flow (e.g., Quataert \& Narayan 1999; Ozel \& Di Matteo 2000). Given a density profile of the form $\rho \propto R^{-3 / 2+p}$ and a temperature profile $T \propto R^{-1}$ (valid at large radii), the bremsstrahlung luminosity is dominated by large radii: $L \propto R^{3} \rho^{2} T^{-1 / 2} \propto R^{2 p+1 / 2}$, assuming photon energies $\lesssim k T(R)$. The resulting spectrum, adding up all radii, is $\Gamma \approx 3 / 2+2 p$, i.e., $\nu L_{\nu} \propto \nu^{1 / 2-2 p}$.

Thus a natural interpretation of the quiescent flux coincident with $\mathrm{Sgr} \mathrm{A}^{*}$ is that it is bremsstrahlung from hot gas in the outer part of the accretion flow that is resolved by Chandra (e.g., Yuan et al. 2001; Quataert 2002). This can account for the size of the source, its lack of variability, and the possible presence of a thermal X-ray line (Baganoff et al. 2003b). The above expression for the spectrum of the thermal emission would imply that $p \approx 1 / 2$ is required to explain the spectrum. There is, however, an important problem with this straightforward interpretation: Chandra spectra are extracted in a $\approx 1$ " region around Sgr $\mathrm{A}^{*}$. This is comparable to the Bondi accretion radius, $R_{a c c}(\S 1)$. It is thus incorrect to assume that observations of the extended emission directly probe the "accretion flow." Instead, they probe the complex "transition region" between the accretion flow and the ambient medium. 

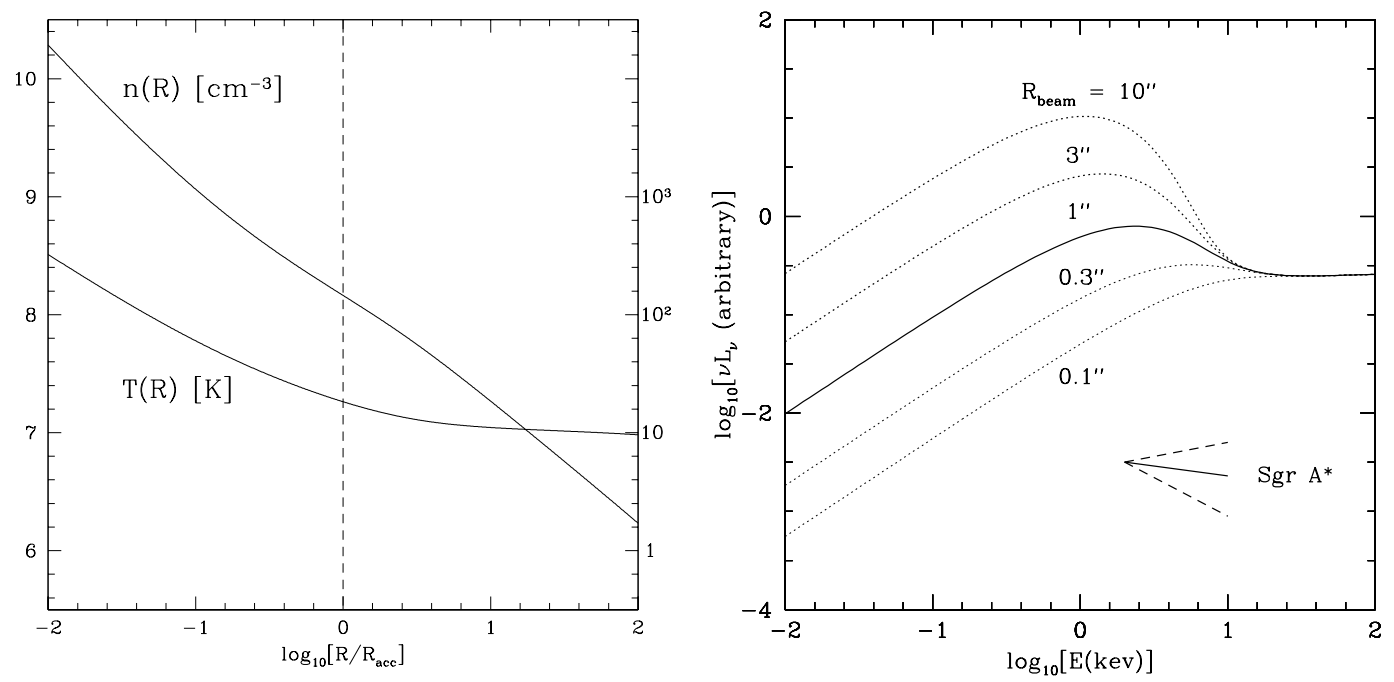

Fig. 3 Left: Observationally motivated models for $n(R)$ and $T(R)$ for hot gas around Sgr A*; models are extrapolated to $R<R_{a c c} \approx 1^{\prime \prime}$ using Bondi accretion. Right: X-ray spectra based on the density and temperature profiles in the left panel. The $R_{\text {beam }} \approx 1^{\prime \prime}$ prediction is consistent with the quiescent X-ray emission coincident with Sgr A*.

Figure 3 illustrates the effects of a finite observing beam relative to the Bondi accretion radius; the left panel shows a toy model for the density and temperature as a function of radius around Sgr A* (in units of $\left.R_{a c c} \approx 1^{\prime \prime}\right)$. The temperature is measured to be $\approx 1 \mathrm{keV}$ at large radii. The density profile on $\gtrsim R_{a c c}$ scales is adjusted to roughly reproduce the radial variation of the observed diffuse $\mathrm{X}$-ray emission (see Quataert 2002 for details). For $R \lesssim R_{a c c}$ gas accretes as a Bondi flow, with asymptotic $\left(R \ll R_{a c c}\right)$ scalings $\rho \propto R^{-3 / 2}$ (i.e., $p=0$ ) and $T \propto R^{-1}$. $^{4}$ Figure 3 b shows the $\mathrm{X}$-ray spectra that would be seen by a telescope with a beam-size $R_{\text {beam }}$. For large beams, $R_{\text {beam }} \approx 10^{\prime \prime}$, the spectrum is very soft and is dominated by the ambient medium that has $T \approx 1 \mathrm{keV}$. For small beams, $R_{\text {beam }} \approx 0.1^{\prime \prime}$, the spectrum is dominated by the accretion flow and is quite hard, consistent with the above scalings. For the case applicable to $\mathrm{Sgr} \mathrm{A}^{*}, R_{\text {beam }} \approx 1^{\prime \prime} \approx R_{a c c}$, the emission is still relatively soft, consistent with the Chandra observations. This is in spite of the fact that the underlying accretion flow produces a hard X-ray spectrum.

The upshot of Figure 3 is that the extended quiescent X-ray source observed by Chandra appears broadly consistent with the emission produced by gas on $R_{a c c} \approx 1^{\prime \prime}$ scales. This is gas in the 'transition region' between the ambient medium and the accretion flow, rather than the accretion flow itself. Using these results to constrain the dynamics of the accreting gas, e.g., the radial density profile $p$, will require (1) better theoretical models for the dynamics of the X-ray emitting gas on $1^{\prime \prime}$ scales, and (2) tighter observational constraints, such as spectra as a function of radius.

\section{Conclusions}

The Galactic Center represents a unique opportunity to probe the dynamics of gas accreting onto a massive black hole, from the 'large' scales on which gas is gravitationally captured by the BH to the 'small' scales close to the BH's horizon. In this review, I have tried to argue that radiatively inefficient accretion flow (RIAF) models can provide a reasonably coherent picture of accretion onto Sgr A* on all of these scales.

\footnotetext{
${ }^{4}$ I chose a Bondi flow for two reasons: (1) Bondi flows predict hard X-ray bremsstrahlung spectra and are thus a good test case for assessing what effect the soft X-ray emitting ambient medium around the BH has on detecting the accretion component, (2) There are no good dynamical models for how rotating RIAFs "match" onto an ambient medium outside $R_{a c c}$.
} 
To summarize: hot gas in the central $\approx 1 \mathrm{pc}$ of the GC is produced by the (shocked) winds from massive stars. This gas is gravitationally captured by the black hole on scales of $R_{a c c} \approx 1^{\prime \prime} \approx 0.04 \mathrm{pc}$. The Chandra detection of an extended soft X-ray component coincident with Sgr A* may be direct evidence for this gravitationally captured gas ( $(3.2)$. The net rate at which gas accretes through the BH's horizon is, however, much less than the canonical Bondi estimate for the rate at which gas is gravitationally captured by the $\mathrm{BH}\left(\dot{M}_{B} \approx 10^{-5} M_{\odot} \mathrm{yr}^{-1}\right)$. The Bondi estimate neglects the angular momentum of the accreting gas, which is likely to be a very poor assumption. Numerical simulations (and analytical models) of rotating RIAFs find that $\dot{M} \ll \dot{M}_{B}(\S 2)$. This conclusion is theoretically attractive because it implies that the radiative efficiency of Sgr A* need not be as low as $\sim 10^{-6}$ as in Bondi and ADAF models (which has always been difficult to reconcile with the expectation that electron heating and acceleration would be important in the collisionless magnetized plasma close to the $\mathrm{BH}$ ). A low accretion rate is also strongly suggested by the detection of linear polarization in the sub-mm emission from Sgr $\mathrm{A}^{*}$ : Faraday rotation constrains the gas density and magnetic field strength close to the BH and argues for $\dot{M} \sim 10^{-8} M_{\odot} \mathrm{yr}^{-1} \ll \dot{M}_{B}$, in good agreement with the inference from RIAF models. Finally, RIAF models with $\dot{M} \sim 10^{-8} M_{\odot} \mathrm{yr}^{-1}$ can explain the basic spectral properties of Sgr A* (see, e.g., Figs. 11 \& 2). In particular, the X-ray flares seen by Chandra may be due to synchrotron or Inverse-Compton emission produced by relativistic electrons accelerated in the inner $\sim 10 R_{S}$ of the accretion flow ( $\left.\$ 3.1\right)$.

It is important to stress that, although $\dot{M} \ll \dot{M}_{B}$ is both theoretically and observationally favored, all of the models considered here are still "radiatively inefficient," and have efficiencies much less than the canonical thin disk value of $10 \%$; e.g., for $\dot{M} \sim 10^{-8} M_{\odot} \mathrm{yr}^{-1}, L \sim 10^{-3} \dot{M} c^{2}$. In fact, all of the physics highlighted in $\S 2$ that suppresses the accretion rate with respect to the Bondi estimate requires a relatively low efficiency and would not operate in a thin disk.

There are several important issues that I have not addressed in this review. To cite two that clearly require further study: (1) are the Chandra flares due to, e.g., reconnection or turbulence in the accretion flow, or are they telling us something more fundamental about the dynamics close to the BH? (2) Both jet and RIAF models can explain the basic spectral properties of Sgr A*. How can we distinguish between these two components, both of which are almost certainly present? E.g., is the linear polarization detection, which requires a relatively coherent magnetic field, consistent with the magnetic field seen in RIAF simulations? Or does it instead require an additional 'jet' component?

Acknowledgements I thank Ramesh Narayan and Feng Yuan for useful discussions. This work is supported by NASA Grant NAG5-12043, NSF Grant AST-0206006, and an Alfred P. Sloan Foundation Fellowship.

\section{References}

[1] Aitken, D. K. et al., 2000, ApJ, 534, L173

[2] Agol, E., 2000, ApJ, 538, L121

[3] Baganoff, F. et al., 2001, Nature, 413, 45

[4] Baganoff, F. et al., 2003a, ApJ, in press

[5] Baganoff, F. et al., 2003b, these proceedings

[6] Beckert, T. \& Falcke, H., 2002, A\&A, 388, 1106

[7] Beckert, T. \& Falcke, H., 2003, these proceedings

[8] Blandford, R.D. \& Begelman, M.C., 1999, MNRAS, 303, L1

[9] Bondi, H., 1952, MNRAS, 112, 195

[10] Bower, G., Wright, M. C. H., Falcke, H., \& Backer, D., 2003, ApJ, in press (astro-ph/0302227)

[11] Falcke, H \& Markoff, S., 2000, A\&A, 362, 113

[12] Ghez, A. et al. 2003, these proceedings

[13] Hawley, J. F. \& Balbus, S. A., 2002, ApJ, 573, 738

[14] Herrnstein, R. M. \& Ho, P. T., 2002, ApJ, 579, L83

[15] Ichimaru, S. 1977, ApJ, 214, 840

[16] Igumenshchev, I. V., \& Abramowicz, M. A. 1999, MNRAS, 303, 309

[17] Igumenshchev, I. V., \& Abramowicz, M. A. 2000, ApJS, 130, 463 
[18] Igumenshchev, I. V., Abramowicz, M. A., \& Narayan, R. 2000, ApJ, 537, L27

[19] Igumenshchev, I. V., Abramowicz, M. A., \& Narayan, R. 2003, ApJ submitted (astro-ph/0301402)

[20] Koratkar, A. \& Blaes, O. 1999, PASP, 111, 1

[21] Krabbe, A., Genzel, R., Drapatz, S., \& Rotaciuc, V., 1991, ApJ, 382, L19

[22] Liu, S. \& Melia, F., 2002, ApJ, 566, L77

[23] Mahadevan, R., 1998, Nature, 394, 651

[24] Manmoto, T., Mineshige, S., \& Kusunose, M., 1997, ApJ, 489, 791

[25] Markoff, S., Falcke, H., Yuan, F., \& Biermann, P. L., 2001, A\&A, 379, L13

[26] Melia, F., 1992, ApJ, 387, L25

[27] Narayan, R. 2002, in Lighthouses of the Universe, ed. M. Gilfanov, \& R. Sunyaev (Heidelberg: Springer-Verlag), in press, astro-ph/0201260

[28] Narayan, R., Igumenshchev, I. V., \& Abramowicz, M. A. 2000, ApJ, 539, 798

[29] Narayan, R., Quataert, E., Igumenshchev, I. V., \& Abramowicz, M. A. 2002, ApJ, 577, 295

[30] Narayan, R. et al., 1998, ApJ, 492, 554

[31] Narayan, R., Yi, I., \& Mahadevan, R., 1995, Nature, 374, 623

[32] Narayan, R., \& Yi, I. 1994, ApJ, 428, L13

[33] Najarro, F. et al., 1997, A\&A, 325, 700

[34] Nayakshin, S., 2003, these proceedings

[35] Ozel, F. \& Di Matteo, T., 2001, ApJ, 563, 276

[36] Ozel, F., Psaltis, D., \& Narayan, R., 2000, ApJ, 541, 234

[37] Quataert, E., 2002, ApJ, 575, 855

[38] Quataert, E., Dorland, W., \& Hammett, G., 2002, ApJ, 577, 524

[39] Quataert, E. \& Gruzinov, A., 1999, ApJ, 520, 248

[40] Quataert, E. \& Gruzinov, A., 2000a, ApJ, 539, 809

[41] Quataert, E. \& Gruzinov, A., 2000b, ApJ, 545, 842

[42] Quataert, E. \& Narayan, R., 1999, ApJ, 520, 298

[43] Rees, M. J., Begelman, M. C., Blandford, R. D., \& Phinney, E. S., 1982, Nature, 295, 17

[44] Ruszkowski, M. \& Begelman, M. C., 2002, ApJ, 573, 485

[45] Schödel, R. et al., 2002, Nature, 419, 694

[46] Shakura, N. I., \& Sunyaev, R. A., 1973, A\&A, 24, 337

[47] Stone, J. M., Pringle, J. E., \& Begelman, M. C. 1999, MNRAS, 310, 1002

[48] Stone, J. \& Pringle, J. E., 2001, MNRAS, 322, 461

[49] Tsuboi, M., Miyazaki, A., \& Tsutsumi, T., 1999, in The Central Parsecs of the Galaxy, ASP Conference Series, ed. Heino Falcke et al., Vol, 186, p. 105

[50] Yuan, F., Markoff, S., \& Falcke, H., 2002, A\&A, 383, 854

[51] Yuan, F., Quataert, E., \& Narayan, R., 2003, in prep.

[52] Zhao, J. et al., 2003, ApJ Letters in press (astro-ph/0302062) 